is now in good health. This was in a case of bronchopneumonia. Other similar cases have strengthened my faith in it under such conditions as described in the paper. Atropin, while it depresses the vagus endings, does not alter the effect of digitalis on the heart muscle directly and is not contraindicated unless other conditions such as auricular fibrillation are present.

Dr. Edward F. WrLls, Chicago: The presence of the form of pulmonary edema which we usually have in mind in pneumonia is not of frequent occurrence but it is a contingency in any case, and for that reason preparation should be made for its possible appearance at the very beginning of treatment. It will not come on early and there is no harm in having things ready. Digitalis-I believe I voice the profession-is a useful remedy in pneumonia, and personally I begin early and continue it throughout the course of the disease, not in very frequent doses, but in amounts producing apparent effect. Strychnin is begun a little later and is given regularly for the special purpose of stimulating the reflexes, the respiratory in particular. Have ready a cylinder of oxygen, which may be of use and certainly can do no harm to the patient, but I do not advise its constant use; have it ready in case of emergency. Full instruc. tions are given to the nurse as to what should be done in the actual appearance, which is usually sudden, of pulmonary edema. These directions consist first in giving at once $1 / 16$ or $1 / 8$ grain of morphin hypodermically. I place but little dependence on atropin, but a small amount may be given, say 1/150 to $1 / 200$ grain; but morphin is the most important remedy in this case. So far as I have seen, the results of treatment depend on the promptness with which these mea sures are taken on the appearance of the edema. The second important advice is that immediately on the advent of edema the mouth and nostrils shall be made the lowest portion of an inclined plane. The whole body should be raised to such degree as will allow any excess fluid in the respiratory tract to escape by the mouth and nostrils. The amount escaping in this manner is sometimes very great. A stimulating enema of glycerin, Epsom salts and water is of secondary importance.

Dr. Walter A. Bastedo, New York: My paper included a paragraph on morphin and also a paragraph on mechanical means for evacuating the fluid. I said morphin had proved successful in paroxysmal edema, but in the complicated edema of pneumonia I believe it should not be employed, as it is dangerous to the respiration, checks expectoration and favors tympanites. I have known edema to be produced by morphin in pneumonia. Like Dr. Wells, I use digitalis early in pneumonia, and this same practice has been adopted at the Rockefeller Institute.

Dr. Wilson's question about atropin versus digitalis is a big one to discuss. Undoubtedly often cardiac failure in pneumonia is the result of the onset of auricular fibrillation or auricular flutter. The impulses going from the auricle to the ventricle cause the ventricle to beat in a disorderly and inefficient manner. In these cases we deal with a disturbance of rhythm, and our desire in the use of digitalis is to prevent the passage of the smaller impulses from the auricle to the ventricle; that is, we desire a degree of heart block so that the ventricle can go on beating efficiently without regard to the erratic beating of the auricle. This is the effect of digitalis in these cases, and it is annulled by atropin. The stimulation of the muscle of the heart has not been proved to be an effect of digitalis that can be elicited satisfactorily in therapeutics; and as a rule the effect on the rhythm is evidently greater than the strengthening effect on the muscle. Furthermore, stimulation of the muscle often has the effect of causing great excitability of some part of the muscle, so that we get extrasystoles without increasing the work of the ventricle. I think, therefore, it is of great importance in pneumonia not to check by the administration of atropin, any possible value of digitalis on the auriculoventricular bundle of His. In regard to $\mathrm{Dr}$. Meltzer's remarks I think that Dr. Welch answered well the arguments against his theory. I am glad that Dr. Meltzer referred to the subject. A disproportion between the right and left ventricles is not an easy thing to determine, and if the heart failure is due to a change in rhythm, as it fre- quently is in pneumonia, both ventricles fail together. The epinephrin question I did not bring up because Dr. Meltzer many years ago established the idea that it was dangerous in any condition that might lead to edema of the lungs. As he mentioned, too, the edema of the lungs from epinephrin occurs usually when you do not want it.

\section{VERTIGO AS A SYMPTOM OF PRIMARY DISEASE OF THE LABYRINTH *}

\section{GEORGE E. SHAMBAUGH, M.D. CHICAGO}

The symptom of vertigo was for the first time associated with disease of the internal ear by Ménière in 1861. Previous to that year, vertigo was considered to be a symptom distinctly of intracranial disease. Ménière published a series of articles on the occurrence of disease of the internal ear presenting symptoms resembling cerebral apoplexy. Eleven cases in all were reported, in which in the absence of any previous ear trouble the patient was suddenly seized with vertigo, associated with tinnitus and deafness. One of his patients died from an unknown cause five days after the attack of vertigo. The macroscopic postmortem examination disclosed a grumous mass filling the semicircular canals. No lesion was detected in the brain or spinal cord. On the basis of these findings, together with the facts regarding disturbance of equilibrium brought out by the experiments which Flourens had previously carried out on the semicircular canals of pigeons, Ménière concluded that the symptoms observed in his patients were the result of hemorrhage into the labyrinth.

Following these publications by Ménière, a great many cases have been reported presenting the characteristic symptoms of sudden onset of vertigo with tinnitus and deafness. Many of these were described as cases of Ménière's disease, and it was assumed that hemorrhage into the labyrinth was responsible for the labyrinth disturbance. The symptoms have appeared quite variant in different cases. In some, a single attack, with sudden onset, threw the patient violently to the ground and produced severe tinnitus with total permanent loss of hearing. In others, there was a long series of attacks, varying widely in severity. In most of these cases each attack registered a distinct progress in an increasing labyrinth deafness. In some, the disturbance of equilibrium, as well as the tinnitus and the deafness, was slight and appeared quite transitory. In still others, the vertigo occurred quite independent of tinnitus or deafness. It became apparent quite early that one cause was not responsible for the symptoms in all of these cases. In some, the symptoms were the result of disturbance in the labyrinth caused by an extension of middle ear infection. In other cases the symptoms were the result of demonstrable intracranial disease. Of those cases in which the disease was primarily in the labyrinth, not infrequently the manner of onset, as well as the transitory character of the disturbance, precluded the possibility that an actual hemorrhage into the labyrinth had given rise to the symptoms.

An effort has been made by some writers to restrict the use of the term "Ménière's disease" to those cases of primary involvement of the labyrinth in which the

* Read before the Section on Laryngology, Otology and Rhinology at the Sixty-Eighth Annual Session of the American Medical Association, New York, June, 1917. 
suddenness of the onset, together with the marked permanent loss in the hearing, seemed to make the diagnosis of an actual hemorrhage into the labyrinth the probable cause. The gradual transition between those cases in which hemorrhage into the labyrinth seemed to account most readily for the symptoms and those in which, from the transitory character of the symptoms, the possibility of an actual hemorrhage was excluded, made it quite impossible to separate a distinct group of cases and assert that these alone were the result of hemorrhage into the labyrinth, and that all the other cases of primary disease of the labyrinth presenting symptoms of attacks of vertigo with tinnitus and disturbed hearing were not caused by hemorrhage.

For these reasons many writers have given up the use of the term "Ménière's disease" for any of these cases, even for those in which it seems certain that a hemorrhage into the labyrinth has given rise to this syndrome, as, for example, when these symptoms occur in cases of pernicious anemia. The term "Ménière syndrome" has, however, come into general use to designate attacks of vertigo when associated with tinnitus and deafness, irrespective of the cause, whether it be from disease of the labyrinth secondary to middle ear infection or to meningitis, or to disease occurring primarily in the labyrinth, or whether it is occasioned by intracranial disease causing disturbance of the central tract of the eighth nerve. This term is an appropriate one by which to designate a characteristic group of symptoms, especially since it serves to perpetuate in otologic literature the name of the physician whose contribution represented a distinct epoch in otology.

Vertigo is now recognized to be as distinctly an aural symptom as is tinnitus, and when present indicates a disturbance of the vestibular nerve, either in its peripheral distribution in the semicircular canals or in its central pathways in the brain. In this paper I shall discuss only those cases of primary disease of the labyrinth producing vertigo.

\section{PHYSIOLOGY}

In order to interpret intelligently the symptoms of vertigo arising from disease of the labyrinth, it is important to keep clearly in mind a few fundamental facts in the physiology of the internal ear. The mechanism in the labyrinth for preserving the body equilibrium, the vestibular apparatus, accomplishes this end largely through the medium of tonus impulses to the skeletal muscles, which keep these muscles in a state of constant stimulation. The tonus impulses from the two labyrinths stimulate for the most part opposing groups of muscles, and since the impulses from both sides are equal, a state of equilibrium is preserved. A sudden alteration in the tonis from one labyrinth disturbs this equilibrium and gives rise to vertigo. The disturbance of equilibrium arising from a sudden disturbance of tonus is always temporary because a compensatory tonus rapidly develops to restore the equilibrium. A gradual destruction of function in one or both labyrinths may proceed even to the point of complete loss of function without ever giving rise to sensations of vertigo.

Keeping these fundamental facts in mind, let us inquire into the various processes which are known to involve the labyrinth primarily and produce the symptom of vertigo. In the first place there can be no doubt that a hemorrhage into the labyrinth will produce the characteristic Ménière syndrome. Hemorrhage into the labyrinth is, however, in all probability a very rare occurrence, whereas vertigo from primary disease of the labyrinth is by no means uncommon. It is indeed doubtful whether the symptoms in any of the cases reported by Ménière were the result of hemorrhage into the labyrinth. Regarding the one case which came to postmortem, there are good reasons for believing that this case was one of epidemic meningitis with extension into the labyrinth. The view that the labyrinth symptoms resulting from large doses of quinin are caused by a congestion or actual hemorrhage into the labyrinth is now given up. In the sar:is way, the Ménière syndrome, which so frequently develops in cases of chronic progressive nerve deafness, is in all probability not often, if ever, the result of hemorrhage in the labyrinth, as was previously supposed. The conditions which are known to produce hemorrhage into the labyrinth include especially such diseases as pernicious anemia, leukemia and purpura hemorrhagica.

Embolism of the labyrinthine artery occurs especially in caisson workers, as the result of gas emboli, and produces in its most characteristic form the apoplectic type of the Ménière syndrome. I have seen a single case in which a blood embolism lodging in the artery of the labyrinth appeared to be the probable cause of a sudden attack of the Ménière syndrome, with a total permanent loss of hearing. The accident occurred during the puerperium, about one week after confinement.

Vertigo is not an infrequent symptom of advanced otosclerosis, in which its occurrence heralds the extension of the spongifying process involving the bony capsule of the labyrinth to the semicircular canals.

Syphilis, both during the secondary and the tertiary stages, often invades the labyrinth, giving rise to attacks of vertigo. Involvement of the labyrinth in hereditary syphilis is also quite frequently observed. In these cases, attacks of vertigo are not at all uncommon, and when present indicate a fresh extension of the disease. Very often in cases of syphilitic degeneration in the labyrinth, in which the involvement of the vestibular mechanism can be demonstrated by means of the turning tests, as well as by caloric stimulation, the process does not cause vertigo. This absence of vertigo is sometimes observed in cases in which, judging from the progress of the deafness, the degeneration appears to be extending quite rapidly. In explanation of the absence of vertigo, it is believed that the loss of function has proceeded so evenly as to allow the development of compensatory tonus to keep pace with the loss of labyrinth tonus. It is only a sudden progress in the degenerative process that enables the disturbance of tonus to give rise to vertigo. This vertigo will take place when the progress of the disease has been comparatively slight, provided only that it develops suddenly.

Neuritis of the eighth nerve is a not infrequent cause of vertigo. This neuritis may be the result of poisoning from drugs, such as quinin, tobacco or alcohol. More often the neuritis is caused by toxins from infectious fevers, such as measles, scarlet fever, diphtheria, influenza, mumps, typhoid and florid tuberculosis. As a rule, the involvement of the vestibular nerve causing attacks of vertigo takes place simultaneously with a degeneration of the cochlear nerves, producing tinnitus and deafness. In some cases the vestibular nerve alone is involved. Here tinnitus and 
deafness are absent, and only the attacks of vertigo indicate the involvement in the internal ear.

By far the most frequent occurrence of vertigo is in association with a chronic degenerative process developing quite independent of syphilis or of the infectious fevers and involving, as a rule, the peripheral neurons of both the cochlear and the vestibular nerves. When the cochlear nerve is involved, the characteristic symptoms are a continuous high pitched tinnitus and a more or less extensive defect in hearing at the upper end of the tone scale. In some cases the vestibular nerve alone is involved. The involvement of the vestibular nerve is not infrequently associated with attacks of vertigo. Such attacks are always an indication of a sudden increase in the degenerative process and are associated with an increase in the tinnitus and the deafness when the cochlear nerve is also involved in the process. It is possible by means of the rotation tests and the caloric stimulation to demonstrate the loss of function taking place in the semicircular canals in these cases. It has been for these cases of primary disease of the labyrinth of unknown origin, presenting the characteristic attacks of the Ménière syndrome, that some would have us preserve the term "Ménière's disease," the assumption being that the distinct attacks are each the result of hemorrhage into the labyrinth.

The characteristic course which these cases take, the chronic progressive nature of the degeneration going on in the labyrinth, the acute exacerbations, often occurring quite suddenly, and resulting in attacks of vertigo associated, as a rule, with tinnitus and deafness, all resemble so closely the course of neuritis in other nerves when caused by chronic foci of infection, that it seems entirely probable that focal infection may be the underlying cause for not a few of these cases of progressive degenerative processes involving the eighth nerve.

The sudden exacerbation producing the characteristic Ménière syndrome can be accounted for exactly as are the exacerbations in any peripheral neuritis of focal origin, as the result of a fresh shower of bacterial emboli liberated from the primary focus and which, for some as yet not entirely accounted for predilection, select the peripheral neurons of the eighth nerve as their target. The varying degree of the severity of the attacks, ranging from a total, even permanent, destruction of function to the slight temporary disturbance sometimes observed, finds here a ready explanation, which was not the condition when we assumed that the severe cases of the Ménière syndrome occurring in primary disease of the internal ear were the result of hemorrhage into the labyrinth, and the milder cases were the result of an angioneurosis. The fact that the degenerative process in the labyrinth may progress without acute exacerbation and therefore without attacks of vertigo is quite in harmony with the chronic neuritis of other nerves.

An additional evidence that focal infection takes a - part in the production of neuritis of the eighth nerve is found in those cases in which the Ménière syndrome is observed in patients suffering from acute articular rheumatism. In the literature there are a number of such cases reported as rheumatism of the internal ear. We now regard acute articular rheumatism as a systemic infection from some primary focus, which very often is located in the faucial tonsil. The occurrence of acute exacerbations is one of the characteristic symptoms developing in cases of acute rheumatism.

\section{SUMMARY}

1. Primary disease of the labyrinth produces attacks of vertigo whenever the vestibular apparatus is involved by an acute process.

2. Hemorrhage into the labyrinth is much less common than was formerly supposed.

3. An embolus lodging in the labyrinthine artery occurs especially in caisson workers, as the result of gas emboli, and gives rise to the characteristic Ménière syndrome.

4. Syphilis in the secondary and tertiary stages, as well as in hereditary syphilis, not infrequently involves the internal ear and gives rise to vertigo.

5. Neuritis of the eighth nerve from overdoses of drugs, such as quinin, tobacco and alcohol, may produce neuritis of the eighth nerve with the Ménière syndrome.

6. Toxic neuritis of the eighth nerve as the result of infectious fevers frequently gives rise to attacks of vertigo.

7. The most frequent occurrence of vertigo is in connection with a primary chronic degenerative process involving the peripheral neurons of the eighth nerve and occurring independent of syphilis or the infectious fevers.

8. The probable cause for many of these cases is to be found in a neuritis of the eighth nerve, as the result of some chronic focus of infection, such as is so frequently seen in chronic disease of the faucial tonsil.

9. Attacks of vertigo occurring in connection with acute articular rheumatism are the result of neuritis of the eighth nerve and a symptom of the systemic infection from some primary focus.

122 South Michigan Avenue.

\section{AURAL VERTIGO OCCURRING IN SUP- PURATI ${ }^{*}$ D DISEASE OF THE MIDDLE EAR*}

\author{
PHILIP D. KERRISON, M.D. \\ NEW YORK
}

I think the majority of experienced otologists will confirm my statement that severe vertigo resulting from middle ear suppuration without labyrinthine involvement is not sufficiently common to call for prolonged discussion here. At least, the distinguishing features of the vertigo in such cases may be more profitably analyzed in connection with special case reports. The following conditions, however, require passing mention:

1. A few cases of chronic middle ear suppuration have been observed or reported in which granulations in the region of the oval window and pressing on the stapes have caused vertigo which has been relieved by careful removal of the granulations. I recall one such case which I saw with Dr. Harmon Smith in which operation later was successfully performed by the late Dr. Sheppard of Brooklyn. The diagnosis of this condition would, in most cases, be tentative prior to operation. It could be determined with some degree of certainty only on $(a)$ the presence of the granulations; $(b)$ retention of fair hearing power; $(c)$ normal reactions to the tests of vestibular irritation, and (d) absence of response to the fistula test. Such cases

* Read before the Section on Laryngology, Otology and Rhinology at the Sixty-Eighth Annual Session of the American Medical Association, New York, June, 1917. 